\title{
LARP1 is regulated by the XIST/miR-374a axis and functions as an oncogene in non-small cell lung carcinoma
}

\author{
ZHIZHENG XU*, JING XU*, HUOQUAN LU, BIN LIN, SHAOPENG CAI, \\ JUN GUO, FARONG ZANG and RUI CHEN \\ Department of Respiratory and Critical Care Medicine, The Second Affiliated Hospital of Zhejiang University \\ School of Medicine (Changxing Branch), Changxing, Huzhou, Zhejiang 313100, P.R. China
}

Received May 6, 2017; Accepted October 3, 2017

DOI: $10.3892 /$ or.2017.6040

\begin{abstract}
La-related protein 1 (LARP1) is a conserved RNA-binding protein and is known to regulate 5'-terminal oligopyrimidine tract (TOP) mRNA translation. Dysregulated LARP1 has been reported to be related to the development of several cancers. However, the exact function and mechanism of LARP1 in non-small cell lung cancer (NSCLC) is largely unknown. In the present study, we found that the mRNA levels of LARP1 were increased in NSCLC cells compared to those in normal control cells. Knockdown of LARP1 inhibited cell proliferation, migration and invasion in NSCLC cells and tumourigenicity in $\mathrm{H} 520$ cells. Both in vitro and in vivo analyses confirmed that STAT3 signalling was inactivated by the knockdown of LARP1. Moreover, LARP1 was identified as a direct target of miR-374a. Overexpression of miR-374a attenuated the promotor effects of LARP1 by inhibiting proliferation, metastasis and STAT3 signalling. Clinically, LARP1 was markedly overexpressed in NSCLC tissues, and upregulated LARP1 was correlated with tumour progression and poor survival. The expression of miR-374a was negatively correlated with the expression of LARP1 in NSCLC tissues. Furthermore, we found that XIST functioned as a competing endogenous RNA to suppress miR-374a, which regulated its downstream target LARP1. In summary, we suggest that the dysfunction of the XIST/miR-374a/LARP1 axis contributes to NSCLC and may serve as a promising therapeutic strategy for treatment.
\end{abstract}

\section{Introduction}

Lung cancer is the leading cause of cancer-related deaths worldwide, particularly in China, and non-small cell lung

Correspondence to: Dr Jing Xu, Department of Respiratory and Critical Care Medicine, The Second Affiliated Hospital of Zhejiang University School of Medicine (Changxing Branch), 66 Taihu Middle Road, Changxing, Huzhou, Zhejiang 313100, P.R. China

E-mail: xujing_doctor@163.com

\section{${ }^{*}$ Contributed equally}

Key words: LARP1, proliferation, invasion, miR-374a, XIST, non-small cell lung carcinoma cancer (NSCLC; including squamous, adenocarcinoma and large cell) accounts for $80 \%$ of all cases $(1,2)$. Despite advances in surgery and radiochemotherapy, the 5-year survival rate of NSCLC patients remains unsatisfactory (1).

La-related protein 1 (LARP1), a member of the LARP family, regulates both mRNA translation and stability (3). In cervical cancer, the knockdown of LARP1 suppressed cell migration, while the overexpression of LARP1 promoted migration through post-transcriptionally regulated genes such as mTOR $(4,5)$. The protein and mRNA levels of LARP1 were upregulated in hepatocellular carcinoma (HCC), and overexpressed LARP1 indicated poor survival in patients (6). Loss of tumour-suppressive miR$26 \mathrm{a} / \mathrm{b}$ increased the invasive ability of prostate cancer cells via targeting of LARP1 (7). However, the biological effect of LARP1 on NSCLC cells is not well understood.

MicroRNAs (miRNAs) are a class of small, non-coding single-stranded RNAs that participate in various biological processes (8). During carcinogenesis, miRNAs have been functionally classified as proto-oncogenes or tumour-suppressor genes and contribute to tumour progression by modulating target genes $(9,10)$. Among these miRNAs, miR-374a functioned as an oncogene in gastric and breast cancer by promoting cell proliferation and invasion $(11,12)$. However, low expression levels of miR-374a in early-stage NSCLC were associated with poor survival in patients (13). miR-374a was significantly decreased in lung adenocarcinoma tissues, and the overexpression of miR-374a resulted in the inhibition of cell proliferation, migration and invasion (14). Thus, the exact function and mechanism of miR-374a in the development of NSCLC warrants further investigation.

It has been widely accepted that the dysregulation of a single miRNA may affect a multitude of mRNAs involved in tumour-related pathways. For example, the activation of STAT3 led to cell proliferation (15), and knockdown of phosphorylated-STAT3 (p-STAT3) inhibited the invasive capacity of pancreatic cancer cells (16). Positive p-STAT3 expression in cervical cancer was associated with lymph node metastasis (17). Both miR-375 and miR-133b suppressed cell growth through the regulation of STAT3 signalling $(18,19)$. All of these findings suggest that the biological behaviour of tumour cells may be regulated by STAT3 signalling activity.

In the present study, we found that silencing of LARP1 inhibited cell proliferation, migration and invasion, as well as 
the downstream STAT3 pathway and epithelial-mesenchymal transition (EMT). Knockdown of LARP1 inhibited tumour growth in vivo. LARP1 was a direct and functional target of miR-374a, which was affected by lncRNA XIST. We revealed that the XIST/miR-374a/LARP1 axis was involved in pulmonary carcinogenesis.

\section{Materials and methods}

Clinical samples and cells. This protocol was approved by the Ethics Committee of the Institutional Review Board at Zhejiang University School of Medicine, and written informed consent was collected before surgery. In total, 84 pairs of primary NSCLC (including adenocarcinoma and squamous cell carcinoma) and adjacent non-tumour tissues were surgically obtained from patients who underwent surgery at the hospital from 2010 to 2012. None of the patients received radiotherapy or chemotherapy prior to surgery. The adjacent non-cancerous tissues were obtained at least $3 \mathrm{~cm}$ away from the tumour margin. All of the samples were snap-frozen in liquid nitrogen and stored at $-80^{\circ} \mathrm{C}$.

NSCLC cell lines (H520, A549, H1299 and H1975) and the lung/bronchial normal epithelial cell line BEAS-2B were obtained from the American Type Culture Collection (ATCC; Manassas, VA, USA), and maintained in RPMI-1640 medium (Gibco, Karlsruhe, Germany) supplemented with $10 \%$ fetal bovine serum (FBS) (Invitrogen, Carlsbad, CA, USA) in a humidified atmosphere of $5 \% \mathrm{CO}_{2}$ at $37^{\circ} \mathrm{C}$.

RNA isolation and quantitative real-time PCR ( $q R T-P C R)$. Total RNA was extracted using TRIzol reagent (Invitrogen) and NanoDrop 2000 (Thermo Fisher Scientific, Inc., Waltham, MA, USA) was used to assess the quality of RNA. Complementary DNA (cDNA) was synthesized using High Capacity cDNA Reverse Transcription kit (ABI, Carlsbad, CA, USA). qRT-PCR was carried out using 10 ng of cDNA with SYBR-Green Master Mix (ABI) on an LightCycler 480 (Roche, Basel, Switzerland). Primers were as follows: LARP1 forward, 5'-CATTAGCTAA GCACATGAAGTG-3' and reverse, 5'-GTACGTACGAATC TTGCAA-3'. For the miRNA assay, the cDNA was synthesized using the One Step PrimeScript miRNA cDNA Synthesis kit (Qiagen, Hilden, Germany), and miRNA was quantified using TaqMan ${ }^{\circledR}$ MicroRNA Assays under the ABI 7900 system (Thermo Fisher Scientific, Inc.). All samples were assessed in triplicates. $\beta$-actin and U6 were used as endogenous controls. Data were analysed using the $2^{-\Delta \Delta \mathrm{Ct}}$ method.

Plasmid construction, oligonucleotides and transfection. To knock down LARP1, we used a lentivirus infection strategy. Lentivirus containing shRNA targeting the LARP1 gene or non-silencing control shRNA were constructed by GeneChem (Shanghai, China). Cells were plated at a density of $30 \%$ and were in good condition before the day of infection. The aforementioned lentivirus was used to treat the H520 and A549 cells according to the manufacturer's protocol.

LARP1 cDNA (XM_005268404) without its 3'-untranslated region (3'-UTR) was amplified by PCR using the primers containing the $M l u \mathrm{I}$ and $K p n \mathrm{I}$ restriction sites and was then inserted into the pcDNA 3.1(+) vector (Invitrogen) to generate the recombinant vector pcLARP1. The primers used were:
5'-GGATTTCCAAGACGCGTCCCATACCTAGCTGCCC-3' (forward) and 5'-CGAGTGGTACCAGAGTGATGGGGCTG GTG-3' (reverse). The empty vector served as a negative control. The miR-374a mimics, inhibitor and corresponding negative controls were purchased from GenePharma, and transfections were performed using Lipofectamine 2000 (Invitrogen).

For the knockdown of XIST, shRNA against lncRNA XIST or scrambled oligonucleotides was ligated into the LV-3 (pGLVH1/GFP + Puro) vector (GenePharma). The 293 cells were co-transfected with Lenti-Pac HIV Expression Packaging Mix and the lentiviral vectors (or the control lentivirus) using Lipofectamine 2000. Lentiviral particles in the supernatant were harvested. The H520 cells were transfected with lentivirus or control virus. The cells were treated with puromycin ( $2 \mu \mathrm{g} / \mathrm{ml}$ ) for 14 days, and GFP-positive cells were selected as sh-XIST and sh-ctrl (control).

Cell proliferation assay. Cell proliferation was determined using the MTT method. Cells $\left(2 \times 10^{3}\right)$ were seeded into 96 -well plates and were maintained at $37^{\circ} \mathrm{C}$. MTT $(100 \mu 1 ; 0.5 \mathrm{mg} / \mathrm{ml}$; Sigma, St. Louis, MO, USA) was added to each well at the indicated time-points (24, 48, 72 or 96 h). Dimethyl sulfoxide (DMSO) $(150 \mu \mathrm{l})$ was used to stop the reaction, followed by measurement of the absorbance at $490 \mathrm{~nm}$.

Wound-healing and Transwell invasion assays. For the wound-healing assay, $1 \times 10^{6}$ cells were seeded into 6 -well plates. A wound was made by scraping the cell monolayer with a $200-\mu 1$ pipette tip. Cell motility was evaluated by observation at intervals of 0 and $24 \mathrm{~h}$. For the cell invasion assay, cells $\left(1 \times 10^{5}\right)$ in serum-free medium were placed into the upper chamber of a 24 -well Transwell chamber $(8-\mu \mathrm{m}$ pore size; Corning, Cambridge, MA, USA) coated with Matrigel (BD Biosciences, San Jose, CA, USA). The bottom chamber was filled with medium containing $10 \%$ FBS. Cells that had invaded through the membrane to the lower surface were fixed, stained and counted.

Luciferase activity assay. Both the wild-type (WT) and mutated (Mut) 3'-UTRs of LARP1 mRNA were subcloned into the XhoI and NotI sites of the psiCHECK-2 vector (Promega, Madison, WI, USA). The mutant constructs were generated using a QuikChange Site-Directed Mutagenesis kit (Stratagene, La Jolla, CA, USA). For the luciferase reporter assay, 293 cells $\left(1 \times 10^{5}\right.$ cells/well) were cultured and co-transfected with $50 \mathrm{nM}$ miR-374a mimics or negative control (Mim-NC), $200 \mathrm{ng}$ of WT or Mut vector, and 2 ng of Renilla luciferase-expressing vector pRL-TK (Promega). Then, $48 \mathrm{~h}$ later, the cells were harvested and assayed using the Dual-Luciferase Reporter Assay system (Promega).

The fragment from XIST containing the predicted miR-374a binding site was amplified by PCR and cloned into a pmirGLO vector (Promega) to form the reporter vector XIST wild-type (XIST-WT). The mutant was generated by mutating the miR-374a seed region binding sequence, which was named XIST-Mut. 293T cells were co-transfected with the pmirGLO vector with either wild-type fragments or mutation fragments and miR-374a using Lipofectamine 2000. Luciferase activity was detected as aforementioned. 
Western blotting. Total protein was extracted by lysing cells in RIPA buffer (Beyotime Institute of Biotechnology, Haimen, China). Cell protein lysates were separated by $10 \%$ sodium dodecyl sulfate-polyacrylamide gel electrophoresis (SDS-PAGE), and then transferred onto a polyvinylidene difluoride (PVDF) membrane (Millipore, Boston, MA, USA). After blocking with $5 \%$ fat-free milk, the membranes were incubated with primary antibodies for LARP1 (ab86359; Abcam, Cambridge, MA, USA), p-STAT3 (Y705, ab76315), cyclin D1 (ab134175), E-cadherin (ab40772) and N-cadherin (ab18203) overnight at $4^{\circ} \mathrm{C}$. PVDF membranes were washed in TBST and incubated with horseradish peroxidase-conjugated secondary antibodies.

Animal experiments. All of the animal protocols were approved by the Institutional Animal Care and Use Committee of Zhejiang University School of Medicine. Male BALB/c nude mice (4-6 weeks old) were purchased from SLAC Laboratory Animal Ltd. (Shanghai, China). The H520 cells $\left(2 \times 10^{6}\right)$ were subcutaneously implanted into the right flank (control shRNA) and left flank (shLARP1) of nude mice. Five weeks later, tumours were harvested, weighed, excised, fixed and embedded in paraffin and sectioned for histological examination of p-STAT3 and Ki67 expression.

Immunohistochemistry (IHC). Mouse tumour tissues were fixed and embedded in paraffin, treated for $2 \mathrm{~h}$ at $65^{\circ} \mathrm{C}$ and then deparaffinized. In addition to standard haematoxylin and eosin (H\&E) staining, the tumour sections were subjected to IHC staining to detect Ki67 (ab92742) and p-STAT3. The sections were incubated with biotinylated secondary antibody, followed by incubation with diaminobenzidine and counterstaining with haematoxylin.

Statistical analysis. Graphical depictions and statistical analyses were conducted using GraphPad Prism 5.0 (GraphPad Software Inc., San Diego, CA, USA) and SPSS 16.0 (SPSS, Inc., Chicago, IL, USA). One-way ANOVA was performed for data involving three or more groups, and sets of two groups were analysed using Student's t-test (two-tailed). The Kaplan-Meier method was used for overall survival analysis. A $\mathrm{P}$-value of $<0.05$ was considered to be statistically significant.

\section{Results}

Silenced LARPI inhibits cell growth and motility. To explore the relationship between LARP1 and the biological behaviour of NSCLC cells, we first detected endogenous LARP1 in NSCLC and BEAS-2B cells. The mRNA levels of LARP1 were higher in NSCLC cells than those in the normal control cells (Fig. 1A). H520 and A549 cells, which expressed relatively high levels of LARP1, were used in the knockdown analysis. Compared with the control cells, shLARP1-transfected H520 cells expressed low levels of LARP1 protein (Fig. 1B). Compared with the negative control group, cell growth was markedly suppressed in the LARP1-silenced group (Fig. 1C). In addition, LARP1 downregulation decreased the migration of H520 and A549 cells compared with that in the control-transfected cells (Fig. 1D). shLARP1-transfected NSCLC cells also exhibited a significant reduction in invasive ability (Fig. 1E).
Knockdown of LARPI inhibits tumour growth in vivo via the STAT3 pathway. As the activated STAT3 pathway is known to be an effector of proliferation and metastasis, we used western blotting to explore the relationship between LARP1 and STAT3 signalling. We found that the protein levels of p-STAT3, cyclin D1 (a cell growth marker), and N-cadherin (an EMT marker) were significantly decreased in shLARP1transfected cells (Fig. 2A), while the protein level of E-cadherin was upregulated. These data indicated that inactivated STAT3 signalling and EMT were involved in shLARP1-induced cell growth and invasion inhibition.

H520 cells infected with shLARP1 were injected into the flanks of nude mice. Mouse tumours derived from the shLARP1transfected cells were significantly smaller than those derived from the vector control (Fig. 2B and C). The weights of the tumours induced by LARP1 downregulation were significantly lower than those induced by the control (Fig. 2D). To determine whether the reduction of tumour growth was a result of the ablation of LARP1, the xenograft tumours were subjected to qRT-PCR for LARP1 mRNA expression. As shown in Fig. 2E, the expression of LARP1 was significantly reduced in shLARP1-treated mice. The expression levels of Ki67 (a cell proliferation marker) and p-STAT3 were decreased in tumours treated with shLARP1 (Fig. 2F).

$L A R P 1$ is a direct target of $m i R-374 a$. We used publicly available databases (TargetScan, microRNA, miRDB) to identify candidate miRNAs that regulated LARP1. miR-374a had a binding site in the 3'-UTR of LARP1 mRNA (Fig. 3A). The luciferase reporter assay revealed that the overexpression of miR-374a significantly suppressed the luciferase activity of the wild-type LARP1 3'-UTR, but failed to affect the mutant 3'-UTR (Fig. 3B). The protein expression of LARP1 was also decreased in miR-374a-overexpressing cells (Fig. 3C).

LARP1 is involved in miR-374a-induced inhibition of cell growth and motility. To ascertain whether miR-374a regulated cell growth and migration through LARP1, we rescued LARP1 expression by introducing the pcDNA3.1-LARP1 plasmid without the 3'-UTR (pcLARP1) or the empty vector (pcDNA3.1) in the presence or absence of ectopic miR374a expression in H520 cells. The expression of LARP1 was confirmed by western blotting (Fig. 3D). The restoration of LARP1 markedly abrogated the miR-374a-induced proliferation, migration and invasion inhibition in H520 cells (Fig. 3E and F). The protein levels of p-STAT3, cyclin D1 and $\mathrm{N}$-cadherin were upregulated in LARP1-overexpressing cells, while E-cadherin was downregulated (Fig. 3G). miR374a mimics led to downregulated p-STAT3, cyclin D1 and $\mathrm{N}$-cadherin and enhanced E-cadherin, which was inconsistent with the phenomenon induced by shLARP1. These data suggested that LARP1 was a functional target of miR-374a.

Upregulated LARPI indicates the advanced phenotype of NSCLC and was negatively associated with miR-374a. The expression of LARP1 was significantly upregulated in NSCLC $(3.567 \pm 1.743)$ tumours compared with that in matched non-tumour tissues $(2.210 \pm 1.217$; $\mathrm{P}<0.001$; Fig. 4A). Upregulated LARP1 was significantly correlated with the advanced tumour-node-metastasis $(\mathrm{TNM})$ stage $(\mathrm{P}=0.001)$, 

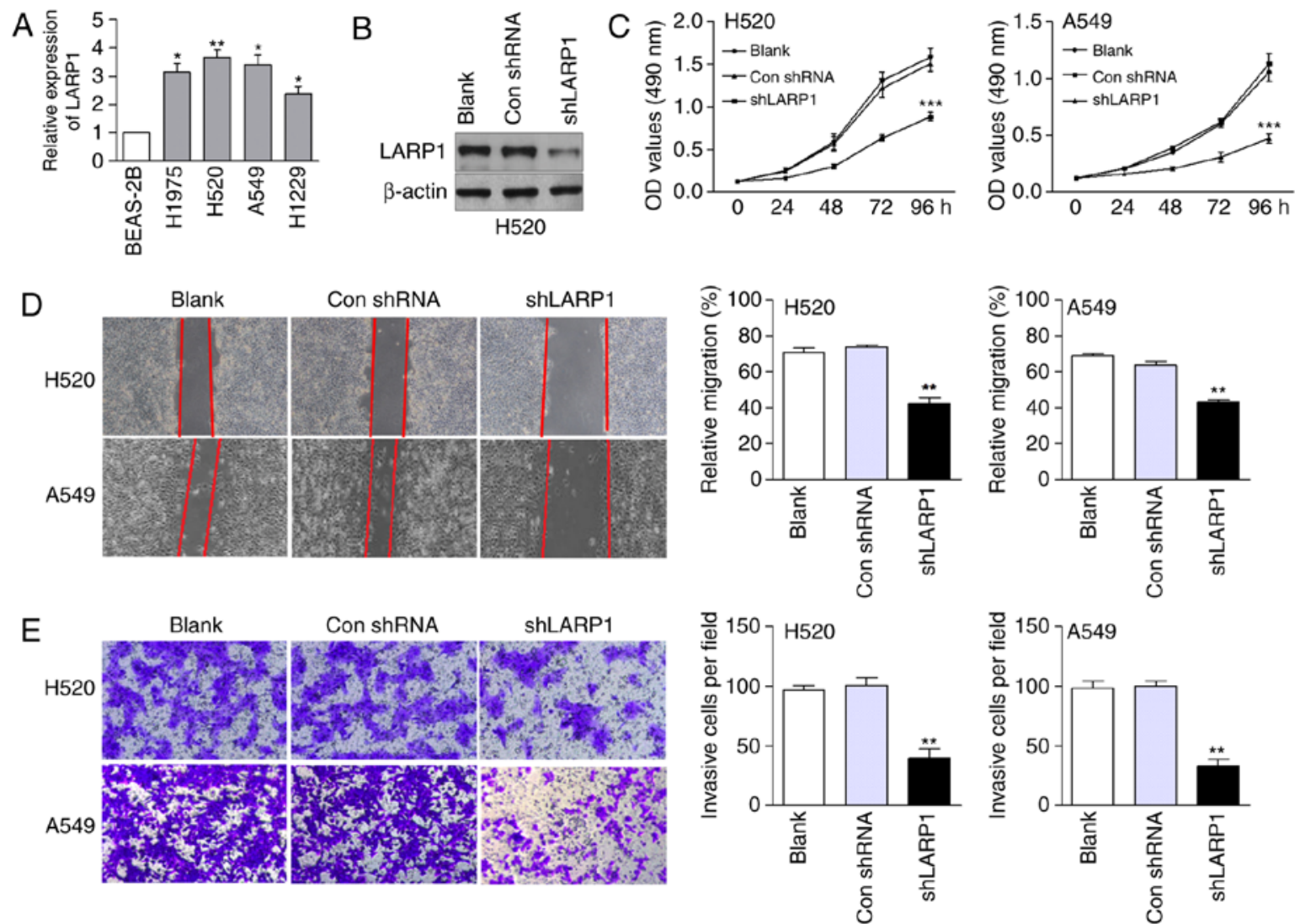

Figure 1. Silenced LARP1 suppresses cell proliferation, migration and invasion. (A) Endogenous LARP1 was higher in NSCLC cells compared to that in BEAS-2B cells. (B) Western blot analysis of the LARP1 infection efficiency after infection with shLARP1 or negative controls (Con shRNA); blank, blank control. (C) MTT assays were used to evaluate the proliferation ability of H520 and A549 cells. Knockdown of LARP1 inhibited (C) migration and (D) invasion in NSCLC cells; ${ }^{*} \mathrm{P}<0.05,{ }^{* *} \mathrm{P}<0.01,{ }^{* * *} \mathrm{P}<0.001$.
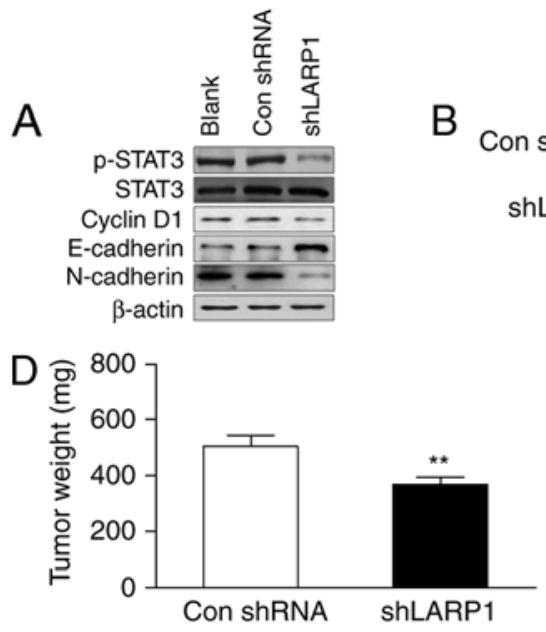
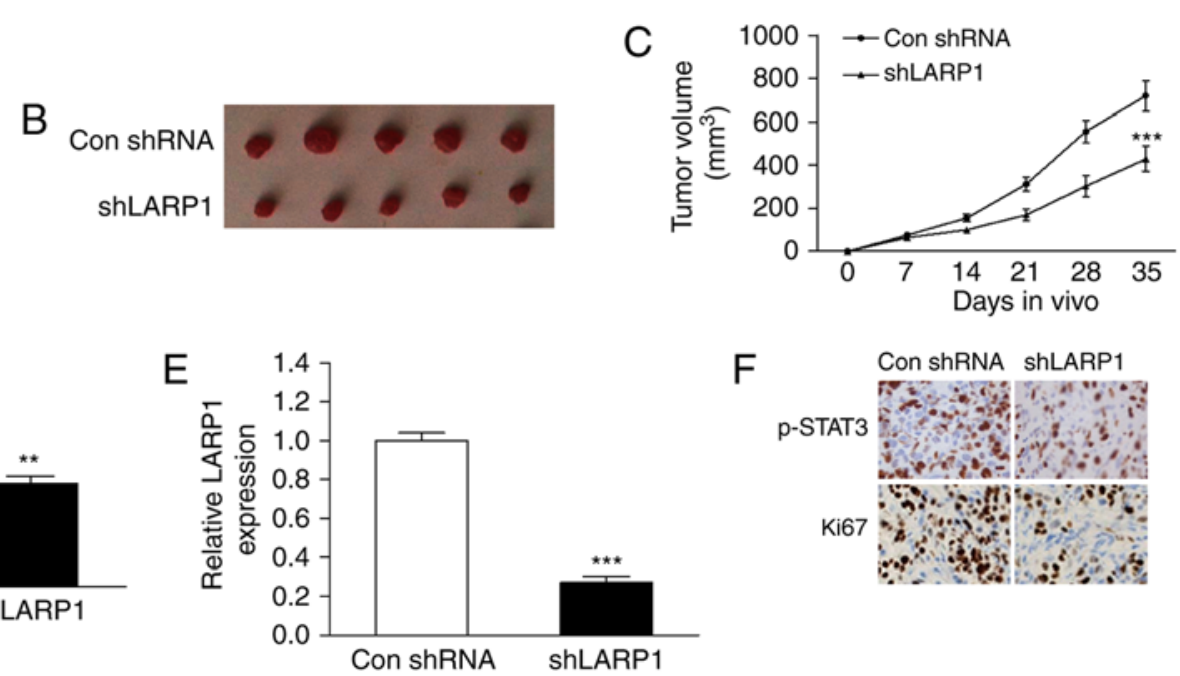

F

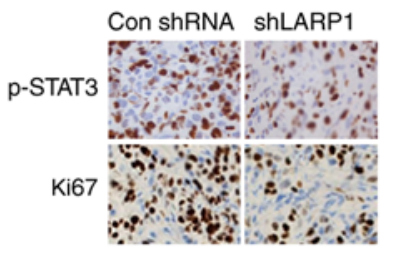

Figure 2. Knockdown of LARP1 reduces tumourigenesis in vivo. (A) STAT3 signalling was inactivated by LARP1 knockdown. (B) Representative tumours are shown. (C) The tumour volume and (D) weight were reduced in LARP1 knockdown tumours. (E) The expression of LARP1 was significantly reduced in shLARP1-treated mice. (F) IHC analysis for p-STAT3 and Ki67 in mouse tumours. Data are expressed as the mean $\pm \mathrm{SD}$; ${ }^{* *} \mathrm{P}<0.01,{ }^{* * *} \mathrm{P}<0.001$.

lymph node $(\mathrm{P}=0.017)$ and distant metastases $(\mathrm{P}=0.039$ Table I). According to the mean level of LARP1 in NSCLC tumours, we separated cases into low or high expression groups. NSCLC patients with low LARP1 expression had better overall survival than those with high LARP1 expression ( $\mathrm{P}=0.022$; Fig. $4 \mathrm{~B}$ ). In addition, to determine the appropriate cut-off for a potential biomarker application, we performed a receiver operating characteristic curve (ROC) analysis. The area under the ROC (AUC) was 0.742 $(\mathrm{P}<0.001)$. The optimal cut-off provided $56 \%$ sensitivity and $82 \%$ specificity for the LARP1 overexpression status as a diagnostic marker for NSCLC (Fig. 4C). 


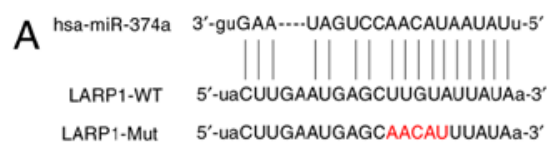

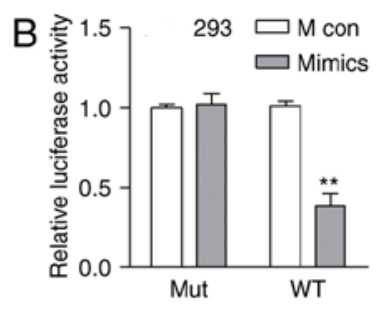
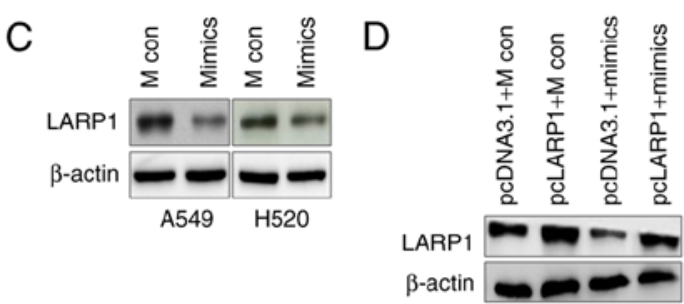

$\mathrm{F}$

H520
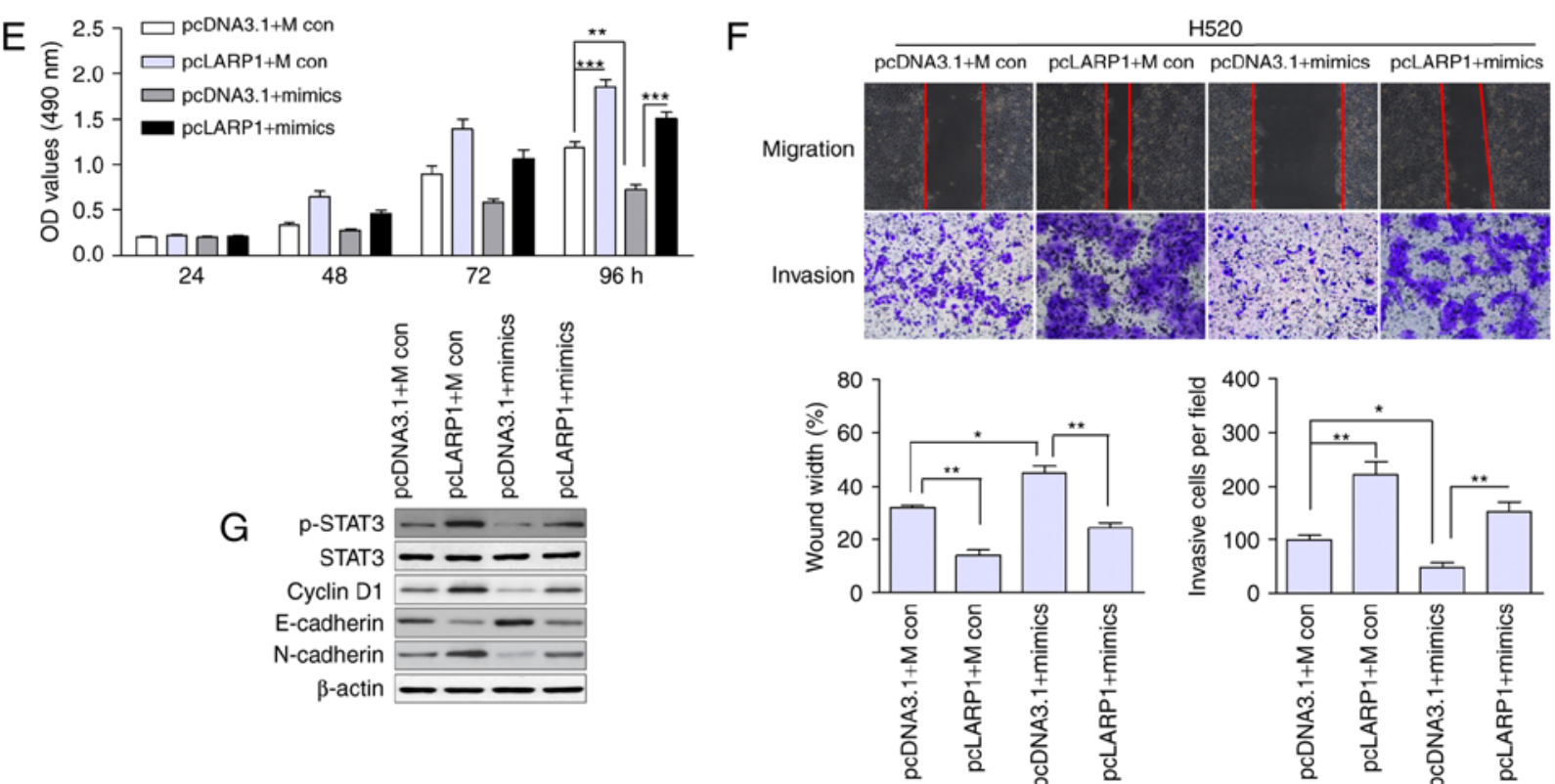

G
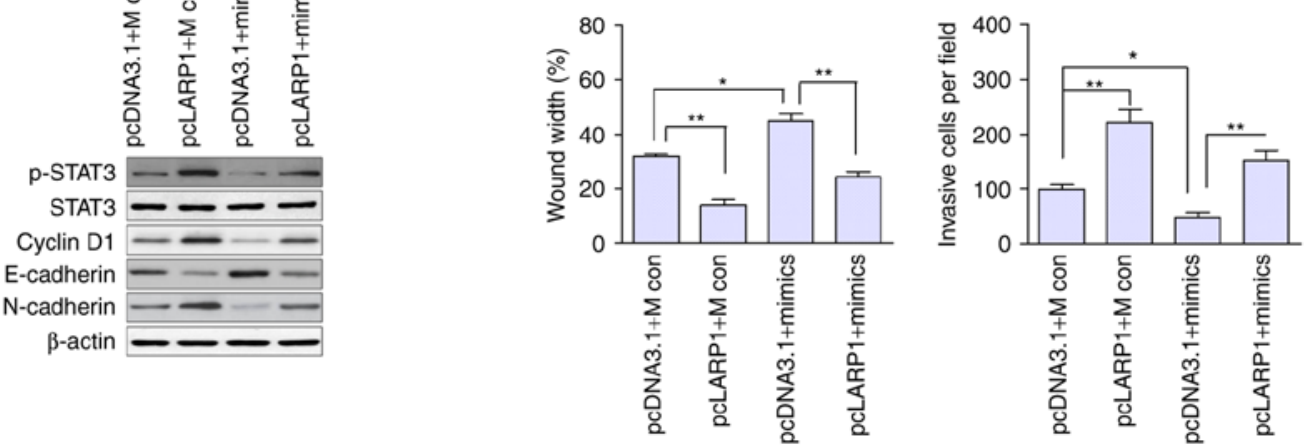

Figure 3. LARP1 is a functional target of miR-374a. (A) There was a putative binding site of miR-374a in the 3'-UTR of LARP1 mRNA. (B) miR-374a inhibited the luciferase activity of the wild-type (WT) 3'-UTR reporter but not the mutant (Mut); M con, mimic control. (C) miR-374a suppressed the protein level of LARP1. (D) The expression levels of LARP1 were examined by western blot analyses using H520 cells that were co-transfected with the miR-374a mimic (or $\mathrm{M}$ con) and pcLARP1 (or pcDNA3.1). (E and F) The promotor effect of LARP1 was attenuated by miR-374a mimics, which inhibited (E) cell proliferation, (F) migration and invasion. (G) STAT3 signalling was activated by LARP1 overexpression or inactivated by miR-374a mimics; ${ }^{*} \mathrm{P}<0.05,{ }^{* * *} \mathrm{P}<0.01,{ }^{* * *} \mathrm{P}<0.001$.
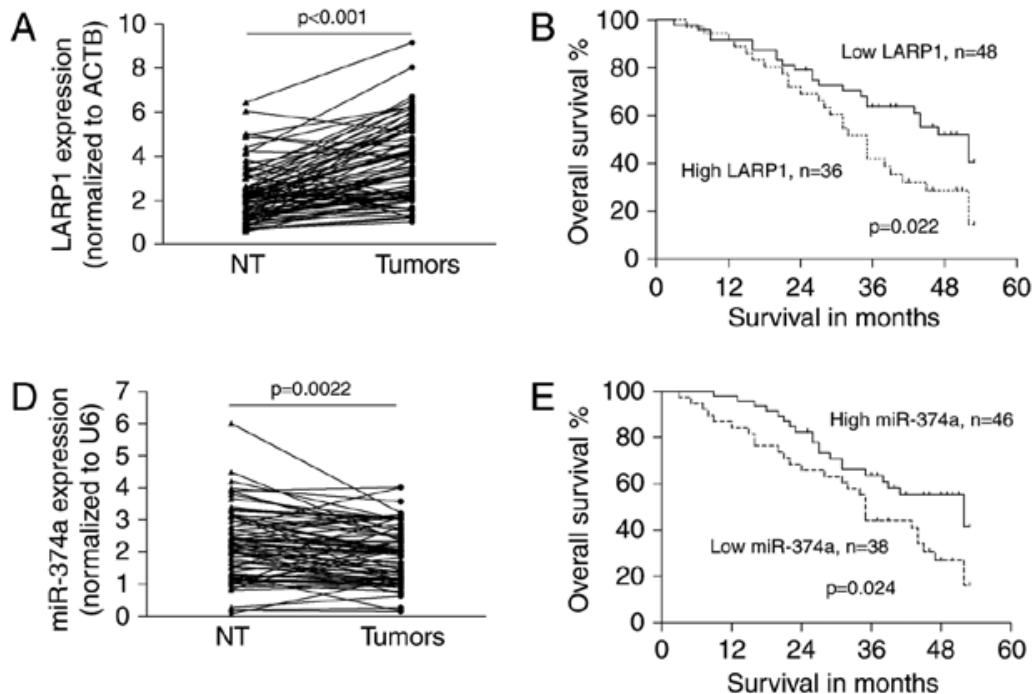
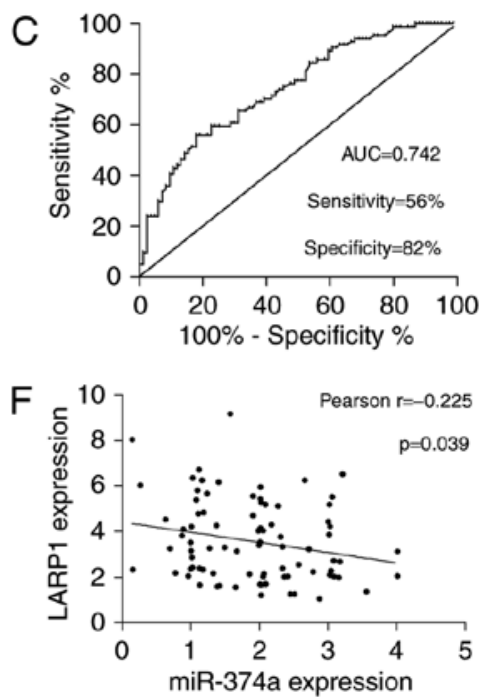

Figure 4. The expression of LARP1 is negatively correlated with miR-374a. (A) The mRNA expression of LARP1 was determined in NSCLC and nontumourous tissues by qRT-PCR; NT, adjacent non-tumour tissues; data are expressed as the means \pm SD. (B) Upregulated LARP1 was related to poor survival in NSCLC cases. (C) The respective area under the receiver operating characteristic curve (ROC) for NSCLC. (D) The expression of miR-374a was decreased in tumours. (E) Downregulated miR-374a indicated poor survival in patients. (F) The expression of LARP1 was inversely associated with miR-374a in tumours.

Moreover, the expression of miR-374a was significantly decreased in primary tumours compared to that in nontumour tissues $(1.892 \pm 0.878$ vs. $2.164 \pm 1.073 ; \mathrm{P}=0.0022$;
Fig. 4D). Downregulated miR-374a was related to advanced tumour stage and distant metastasis $(\mathrm{P}=0.012$, and 0.005 , respectively; Table I). The mean level of miR-374a in NSCLC 
Table I. Correlations between the expression of LARP1/miR-374a and clinicopathological characteristics of NSCLC patients .

\begin{tabular}{|c|c|c|c|c|c|}
\hline Characteristics & Cases $(n=84)$ & LARP1 expression & P-value & miR-374a expression & P-value \\
\hline Sex & & & $0.396^{\mathrm{a}}$ & & $0.516^{\mathrm{a}}$ \\
\hline Female & 30 & $3.445 \pm 1.661$ & & $1.839 \pm 0.964$ & \\
\hline Male & 54 & $3.634 \pm 1.799$ & & $1.921 \pm 0.835$ & \\
\hline Age (years) & & & $0.754^{\mathrm{a}}$ & & $0.779^{\mathrm{a}}$ \\
\hline$<60$ & 39 & $3.540 \pm 1.779$ & & $2.075 \pm 0.934$ & \\
\hline$\geq 60$ & 45 & $3.590 \pm 1.732$ & & $1.734 \pm 0.805$ & \\
\hline Smoking status & & & $0.089^{\mathrm{b}}$ & & $0.152^{\mathrm{b}}$ \\
\hline Never & 32 & $3.330 \pm 1.445$ & & $1.795 \pm 1.064$ & \\
\hline Current smoker & 42 & $3.626 \pm 2.028$ & & $2.000 \pm 0.939$ & \\
\hline Former smoker & 10 & $4.075 \pm 1.267$ & & $1.660 \pm 0.581$ & \\
\hline Differentiation & & & $0.263^{\mathrm{a}}$ & & $0.686^{\mathrm{a}}$ \\
\hline Well & 29 & $3.319 \pm 1.526$ & & $1.894 \pm 1.746$ & \\
\hline Moderate, poor & 55 & $3.697 \pm 1.847$ & & $1.891 \pm 0.947$ & \\
\hline TNM stage & & & $0.001^{\mathrm{a}}$ & & $0.012^{\mathrm{a}}$ \\
\hline $\mathrm{I}, \mathrm{II}$ & 37 & $2.718 \pm 1.188$ & & $2.220 \pm 0.883$ & \\
\hline III,IV & 47 & $4.235 \pm 1.829$ & & $1.633 \pm 0.792$ & \\
\hline LNM status & & & $0.017^{\mathrm{a}}$ & & $0.096^{\mathrm{a}}$ \\
\hline Negative & 23 & $2.557 \pm 1.025$ & & $2.285 \pm 0.980$ & \\
\hline Positive & 61 & $3.948 \pm 1.811$ & & $1.744 \pm 0.796$ & \\
\hline Distant metastasis & & & $0.039^{\mathrm{a}}$ & & $0.005^{\mathrm{a}}$ \\
\hline No & 78 & $3.407 \pm 1.656$ & & $1.986 \pm 0.833$ & \\
\hline Yes & 6 & $5.645 \pm 1.624$ & & $0.667 \pm 0.416$ & \\
\hline Histological type & & & $0.436^{\mathrm{a}}$ & & $0.644^{\mathrm{a}}$ \\
\hline $\mathrm{SCC}$ & 53 & $3.569 \pm 1.781$ & & $1.798 \pm 0.840$ & \\
\hline $\mathrm{AC}$ & 31 & $3.564 \pm 1.705$ & & $2.052 \pm 0.933$ & \\
\hline
\end{tabular}

Never, no smoking history; former smoker, stopped smoking over 1 year; LARP1, La-related protein 1; NSCLC, non-small cell lung cancer; TNM, tumour-node-metastasis; LNM, lymph node metastasis; SCC, squamous cell carcinoma; AC, adenocarcinoma; ${ }^{a}$ Mann-Whitney U test; ${ }^{\mathrm{b}}$ Kruskal-Wallis test.

tissues was used as a threshold to classify NSCLC cases into low or high expression groups; and low expression of miR-374a indicated poor survival in patients $(\mathrm{P}=0.024$; Fig. $4 \mathrm{E})$. There was an inverse correlation between the expression of miR-374a and LARP1 in tumour tissues (Pearson $\mathrm{r}=-0.225$; $\mathrm{P}=0.039$; Fig. 4F), suggesting that the miR-374a/LARP1 regulatory pathway was related to the progression of NSCLC.

Reciprocal repression of IncRNA XIST and miR-374a expression. Using the starBase v2.0 database, miR-374a was found to potentially bind to XIST (Fig. 5A). The qRT-PCR assay showed that miR-374a expression was increased in the sh-XIST group (Fig. 5B). We cloned the predicted miR-374a binding site of XIST (XIST-WT) and a mutated binding site (XIST-Mut) into a reporter plasmid. The results showed that co-transfection of miR-374a mimics and XIST-WT decreased the luciferase activity (Fig. 5C). As shown in Fig. 5D, XIST expression was reduced in cells treated with miR-374a mimics, whereas the expression in cells treated with the miR-374a inhibitor was increased. Finally, we determined whether XIST can regulate the expression of LARP1. Knockdown of XIST reduced the mRNA and protein levels that were increased by the miR-374a inhibitor (Fig. 5E and F). Functionally, the knockdown of XIST significantly inhibited proliferation in H520 cells and abolished the miR-374a inhibition-induced increase in cell proliferation (Fig. 5G). Likewise, knockdown of XIST suppressed the migration and invasion ability increased by miR-374a inhibition in H520 cells (Fig. 5H and I). These results indicated that XIST regulates miR-374a to modulate cell viability and motility in NSCLC cells. We also measured the expression of XIST in H1975 cells derived from female and in H520, A549 and H1299 cells derived from male. There was no significant difference between the expression of XIST in female and male cancer cells (data not shown).

\section{Discussion}

LARP1 has been reported as a key regulator of the mTOR signalling pathway and is involved in cancer progression (5). The mRNA and protein levels of LARP1 were increased in hepatocellular carcinoma (HCC), and upregulated LARP1 indicated large tumour sizes and poor survival (6). In 

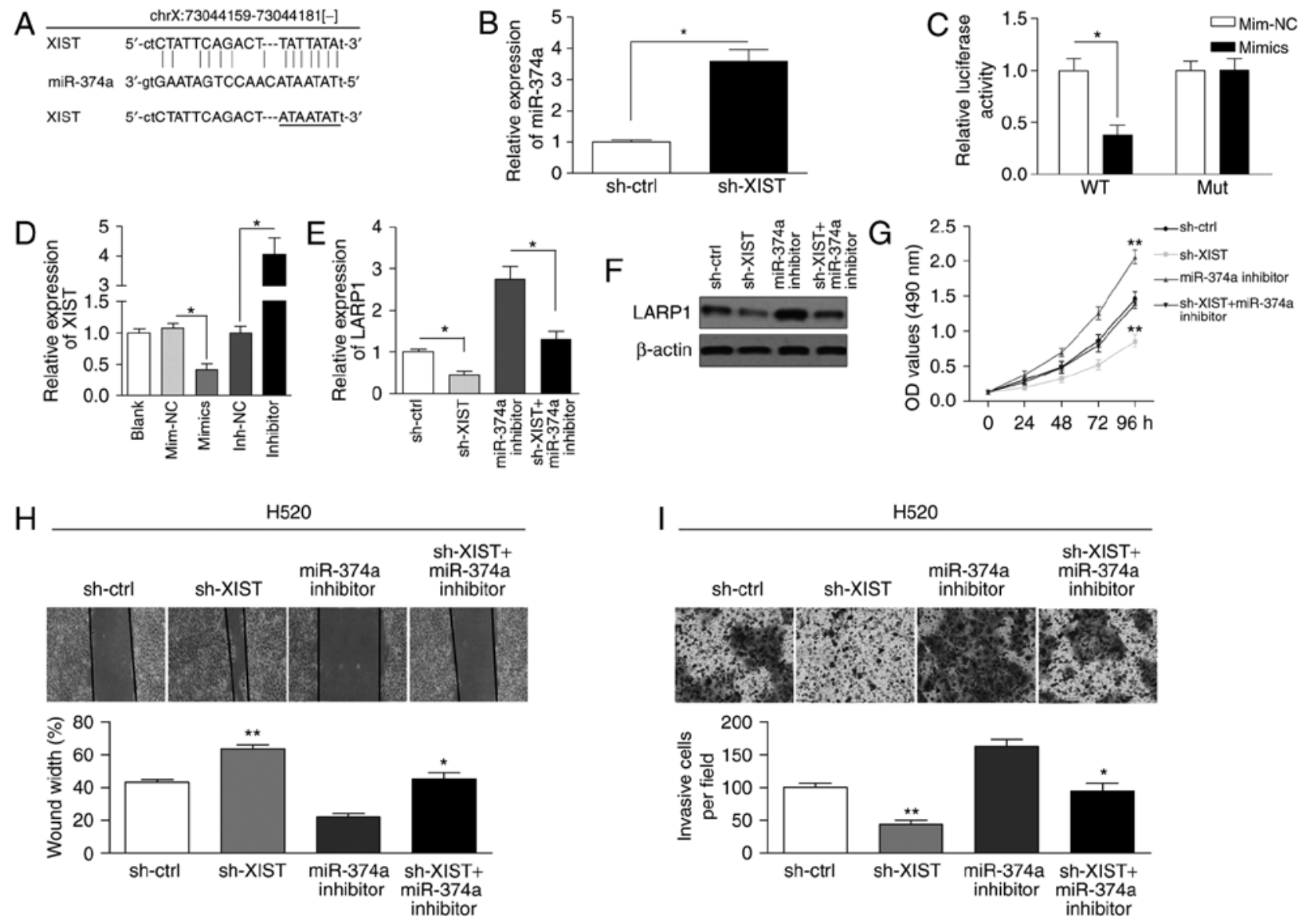

Figure 5. Reciprocal suppression of lncRNA XIST and miR-374a in H520 cells. (A) The binding sites of miR-374a on XIST. (B) miR-374a expression was increased in sh-XIST cells when compared with that in sh-ctrl cells. (C) Luciferase activity of reporters containing XIST-WT or TUG1-Mut sequences in H520 cells transfected with miR-374a mimic or control (Mim-NC). (D) The expression of XIST was decreased by miR-374a mimics, but increased by the inhibitor. (E) The mRNA and (F) protein levels of LARP1 were decreased by shXIST and restored by the miR-374a inhibitor. (G) Cell viability was determined by MTT assays. (H and I) Wound healing and Transwell invasion assays were used to assess $(\mathrm{H})$ the migration and (I) invasion of $\mathrm{H} 520$ cells; ${ }^{*} \mathrm{P}<0.05,{ }^{* *} \mathrm{P}<0.01$.

colorectal cancer (CRC), the increased expression of LARP1 was associated with $\mathrm{T}, \mathrm{N}$ and $\mathrm{M}$ stage and was independently correlated with patient survival (20). In the present study, we found similar results. LARP1 was overexpressed in NSCLC tumours and increased LARP1 was related to cancer progression and worse survival. Further studies with larger groups of patients are needed to confirm these findings. By analysing data sets from Oncomine (www.oncomine.org), we found that the expression of LARP1 was increased in lung and bladder cancer, CRC and HCC, as well as in myeloma (data not shown), supporting the role of LARP1 as an important oncogene that may be commonly involved in various types of human malignancies. In addition, since LARP1 could distinguish normal tissues from tumours with $82 \%$ specificity in the present study, it may be used as a novel diagnostic marker.

For the biological function of LARP1, we observed the inhibitory effects of LARP1 silencing on cell growth, migration, invasion and tumourigenicity. In previous studies, LARP1 promoted cervical cancer cell migration and invasion, while knockdown of LARP1 attenuated the invasive ability of prostate cancer cells $(5,7)$. LARP1 partially abolished miR-374a-induced suppression of cell growth, a finding that was consistent with previous studies revealing that LARP1 promoted cell proliferation (21). Thus, LARP1 functioned as an oncogene by promoting cell growth and motility, which was consistent with the observations revealing that LARP1 was related to metastasis in clinical samples. Collectively, our findings expanded on the knowledge of LARP1 in NSCLC and suggested the cancer-promoting activity of LARP1.

Tumour metastasis has been widely accepted as a major obstacle to successful cancer treatment and ultimately leads to poor prognosis in NSCLC patients (22). Previous studies have documented that constitutive STAT3 activation contributed to metastasis in lung cancer $(23,24)$. Additionally, activated STAT3 signalling has been observed in breast cancer and HCC $(25,26)$. In the present study, we found that p-STAT3 proteins were attenuated in cells and mouse tumours with LARP1 silencing. Upregulated LARP1 in cells activated the expression of p-STAT3, which could be reversed by miR-374a overexpression. miR-374a inhibited cell proliferation and motility, as well as protein levels of cyclin D1 and N-cadherin. All these results revealed that miR-374a may influence the biological behaviour of NSCLC cells by regulating LARP1 and downstream STAT3 signalling.

Accumulating evidence has indicated that dysregulation of miRNAs promotes the pathogenesis of human malignancies. Herein, we found that downregulated miR-374a was associated with advanced tumour stage and poor survival. Similarly, downregulated miR-374a was significantly associated with poor survival in lung cancer patients (14). In addition, 
miR-374a abrogated the promotor effect of LARP1 on cell proliferation, migration and invasion in vitro, suggesting a tumour-suppressive role for miR-374a in NSCLC. A previous study reported that the expression level of miR-374a was suppressed in invasive adenocarcinoma (27), and overexpression of miR-374a inhibited cell growth and metastasis (15). All these studies suggest that miR-374a may function as a tumour suppressor and that the miR-374a/LARP1 axis may play a role in pulmonary tumourigenesis.

Recently, numerous studies have shown that lncRNAs can act as ceRNA for miRNAs; they function as molecular sponges to competitively inhibit miRNAs. For example, XIST modulated gastric cancer development by acting as a molecular sponge of miR-101 to regulate EZH2 expression (28). XIST could bind to miR-320b and suppress miR-320b, which was involved in the dysregulation of RAP2B (29). From a publicly available database, we found that XIST could potentially bind to miR-374a. A dual-luciferase reporter assay was used to ascertain this relationship. The results revealed that the luciferase activity of the reporter containing XIST-WT was decreased in cells transfected with the miR-374a mimic. XIST and miR-374a could inhibit each other. Furthermore, the miR-374a inhibitor restored the inhibition effect of sh-XIST on the expression of LARP1. Former studies demonstrated that XIST contributed to NSCLC via the targeting of different miRNAs, such as miR-449a and miR-186 $(30,31)$, implying that XIST may regulate the progression of NSCLC by targeting multiple miRNAs. Herein, we found that the knockdown of XIST exerted a tumour-suppressive role by inhibiting cell growth, migration and invasion, which was inconsistent with previous studies $(28,32)$. However, XIST cannot only function as an oncogene in tumourigenesis, but also function as a master regulator of X chromosome inactivation (XCI) initiation. There is no corresponding evidence to show that LARP1 is linked to XCI. However, mmu-miR-374 was found to be mapped adjacent to XIST that were predominantly expressed in female blastocysts (33). LncRNA FTX was reported as a positive regulator of XIST and to be involved in XCI (34). Notably, FTX suppressed cell growth, metastasis, Wnt/ $\beta$ catenin signalling by competitively sponging miR-374a. Thus, we speculated that miR-374a may be related with XCI by interacting with XIST and/or FTX.

In conclusion, knockdown of LARP1 inhibited proliferation and motility in NSCLC cells, phenomena that contributed to NSCLC progression. Upregulated LARP1 may be used as a potential prognostic and diagnostic factor. Our findings highlighted that LARP1 was post-transcriptionally regulated by miR-374a, and the XIST/miR-374a/LARP1 axis provided novel insight into the molecular mechanisms of NSCLC oncogenesis.

\section{Acknowledgements}

The present study was supported by the Foundation of Huzhou Science and Technology Bureau Project (grant no. 2015GY33).

\section{References}

1. Siegel R, Naishadham D and Jemal A: Cancer statistics, 2013. CA Cancer J Clin 63: 11-30, 2013.
2. Chen WQ, Zheng RS, Zhang SW, Li N, Zhao P, Li GL, Wu LY and He J: Report of incidence and mortality in china cancer registries, 2008. Chin J Cancer Res 24: 171-180, 2012.

3. Stavraka C and Blagden S: The La-related proteins, a family with connections to cancer. Biomolecules 5: 2701-2722, 2015.

4. Burrows C, Abd Latip N, Lam SJ, Carpenter L, Sawicka K, Tzolovsky G, Gabra H, Bushell M, Glover DM, Willis AE, et al: The RNA binding protein Larp1 regulates cell division, apoptosis and cell migration. Nucleic Acids Res 38: 5542-5553, 2010.

5. Mura M, Hopkins TG, Michael T, Abd-Latip N, Weir J, Aboagye E, Mauri F, Jameson C, Sturge J, Gabra H, et al: LARP1 post-transcriptionally regulates mTOR and contributes to cancer progression. Oncogene 34: 5025-5036, 2015.

6. Xie C, Huang L, Xie S, Xie D, Zhang G, Wang P, Peng L and Gao Z: LARP1 predict the prognosis for early-stage and AFP-normal hepatocellular carcinoma. J Transl Med 11: 272, 2013.

7. Kato M, Goto Y, Matsushita R, Kurozumi A, Fukumoto I, Nishikawa R, Sakamoto S, Enokida H, Nakagawa M, Ichikawa T, et al: MicroRNA-26a/b directly regulate La-related protein 1 and inhibit cancer cell invasion in prostate cancer. Int J Oncol 47: 710-718, 2015.

8. Li XJ, Luo XQ, Han BW, Duan FT, Wei PP and Chen YQ: MicroRNA-100/99a, deregulated in acute lymphoblastic leukaemia, suppress proliferation and promote apoptosis by regulating the FKBP51 and IGF1R/mTOR signalling pathways. Br J Cancer 109: 2189-2198, 2013.

9. Ambs S, Prueitt RL, Yi M, Hudson RS, Howe TM, Petrocca F, Wallace TA, Liu CG, Volinia S, Calin GA, et al: Genomic profiling of microRNA and messenger RNA reveals deregulated microRNA expression in prostate cancer. Cancer Res 68: 6162-6170, 2008.

10. Munker R and Calin GA: MicroRNA profiling in cancer. Clin Sci 121: 141-158, 2011

11. Xu X, Wang W, Su N, Zhu X, Yao J, Gao W, Hu Z and Sun Y: miR-374a promotes cell proliferation, migration and invasion by targeting SRCIN1 in gastric cancer. FEBS Lett 589: 407-413, 2015.

12. Cai J, Guan H, Fang L, Yang Y, Zhu X, Yuan J, Wu J and Li M: MicroRNA-374a activates Wnt/ $\beta$-catenin signaling to promote breast cancer metastasis. J Clin Invest 123: 566-579, 2013.

13. Võsa U, Vooder T, Kolde R, Fischer K, Välk K, Tõnisson N, Roosipuu R, Vilo J, Metspalu A and Annilo T: Identification of miR-374a as a prognostic marker for survival in patients with early-stage nonsmall cell lung cancer. Genes Chromosomes Cancer 50: 812-822, 2011.

14. Wu H, Liu Y, Shu XO and Cai Q: MiR-374a suppresses lung adenocarcinoma cell proliferation and invasion by targeting TGFA gene expression. Carcinogenesis 37: 567-575, 2016.

15. Riemenschneider MJ, Betensky RA, Pasedag SM and Louis DN: AKT activation in human glioblastomas enhances proliferation via TSC2 and S6 kinase signaling. Cancer Res 66: 5618-5623, 2006.

16. Qiu Z, Huang C, Sun J, Qiu W, Zhang J, Li H, Jiang T, Huang K and Cao J: RNA interference-mediated signal transducers and activators of transcription 3 gene silencing inhibits invasion and metastasis of human pancreatic cancer cells. Cancer Sci 98: 1099-1106, 2007.

17. Takemoto S, Ushijima K, Kawano K, Yamaguchi T, Terada A, Fujiyoshi N, Nishio S, Tsuda N, Ijichi M, Kakuma T, et al: Expression of activated signal transducer and activator of transcription-3 predicts poor prognosis in cervical squamous-cell carcinoma. Br J Cancer 101: 967-972, 2009.

18. Wei R, Yang Q, Han B, Li Y, Yao K, Yang X, Chen Z, Yang S, Zhou J, Li M, et al: microRNA-375 inhibits colorectal cancer cells proliferation by downregulating JAK2/STAT3 and MAP3K8/ERK signaling pathways. Oncotarget 8: 16633-16641, 2017.

19. Cheng $\mathrm{N}$ and Wang GH: miR-133b, a microRNA targeting S1PR1, suppresses nasopharyngeal carcinoma cell proliferation. Exp Ther Med 11: 1469-1474, 2016.

20. Ye L, Lin ST, Mi YS, Liu Y, Ma Y, Sun HM, Peng ZH and Fan JW: Overexpression of LARP1 predicts poor prognosis of colorectal cancer and is expected to be a potential therapeutic target. Tumour Biol 37: 14585-14594, 2016.

21. Tcherkezian J, Cargnello M, Romeo Y, Huttlin EL, Lavoie G, Gygi SP and Roux PP: Proteomic analysis of cap-dependent translation identifies LARP1 as a key regulator of 5'TOP mRNA translation. Genes Dev 28: 357-371, 2014. 
22. Sandler AB: Molecular targeted agents in non-small-cell lung cancer. Clin Lung Cancer 5 (Suppl 1): S22-S28, 2003.

23. Song L, Rawal B, Nemeth JA and Haura EB: JAK1 activates STAT3 activity in non-small-cell lung cancer cells and IL-6 neutralizing antibodies can suppress JAK1-STAT3 signaling. Mol Cancer Ther 10: 481-494, 2011.

24. Chiu HC, Chou DL, Huang CT, Lin WH, Lien TW, Yen KJ and Hsu JT: Suppression of Stat 3 activity sensitizes gefitinibresistant non small cell lung cancer cells. Biochem Pharmacol 81: 1263-1270, 2011

25. Lee C, Dhillon J, Wang MY, Gao Y, Hu K, Park E, Astanehe A Hung MC, Eirew P, Eaves CJ, et al: Targeting YB-1 in HER-2 overexpressing breast cancer cells induces apoptosis via the mTOR/STAT3 pathway and suppresses tumor growth in mice. Cancer Res 68: 8661-8666, 2008.

26. Lin $\mathrm{YH}, \mathrm{Wu} \mathrm{MH}$, Liao CJ, Huang YH, Chi HC, Wu SM, Chen CY, Tseng YH, Tsai CY, Chung IH, et al: Repression of microRNA-130b by thyroid hormone enhances cell motility. J Hepatol 62: 1328-1340, 2015.

27. Sato T, Shiba-Ishii A, Kim Y, Dai T, Husni RE, Hong J, Kano J, Sakashita S, Iijima T and Noguchi M: miR-3941: A novel microRNA that controls IGBP1 expression and is associated with malignant progression of lung adenocarcinoma. Cancer Sci 108: 536-542, 2017

28. Chen DL, Ju HQ, Lu YX, Chen LZ, Zeng ZL, Zhang DS, Luo HY, Wang F, Qiu MZ, Wang DS, et al: Long non-coding RNA XIST regulates gastric cancer progression by acting as a molecular sponge of miR-101 to modulate EZH2 expression. J Exp Clin Cancer Res 35: 142, 2016.
29. Lv GY, Miao J and Zhang XL: Long non-coding RNA XIST promotes osteosarcoma progression by targeting Ras-related protein RAP2B via miR-320b. Oncol Res: Apr 12, 2017 (Epub ahead of print). doi: 10.3727/096504017X14920318811721.

30. Zhang YL, Li XB, Hou YX, Fang NZ, You JC and Zhou QH: The lncRNA XIST exhibits oncogenic properties via regulation of miR-449a and Bcl-2 in human non-small cell lung cancer. This article has been corrected since Advanced Online Publication, and an erratum is also printed in this issue. Acta Pharmacol Sin 38: 371-381, 2017.

31. Wang H, Shen Q, Zhang X, Yang C, Cui S, Sun Y, Wang L, Fan X and Xu S: The long non-coding RNA XIST controls non-small cell lung cancer proliferation and invasion by modulating miR-186-5p. Cell Physiol Biochem 41: 2221-2229, 2017.

32. Yao Y, Ma J, Xue Y, Wang P, Li Z, Liu J, Chen L, Xi Z, Teng H, Wang Z, et al: Knockdown of long non-coding RNA XIST exerts tumor-suppressive functions in human glioblastoma stem cells by up-regulating miR-152. Cancer Lett 359: 75-86, 2015.

33. Kobayashi S, Totoki Y, Soma M, Matsumoto K, Fujihara Y, Toyoda A, Sakaki Y, Okabe M and Ishino F: Identification of an imprinted gene cluster in the X-inactivation center. PLoS One 8: e71222, 2013

34. Chureau C, Chantalat S, Romito A, Galvani A, Duret L, Avner P, Rougeulle C, Okabe M and Ishino F: Ftx is a non-coding RNA which affects Xist expression and chromatin structure within the X-inactivation center region. Hum Mol Genet 20: 705-718, 2011. 